\title{
Results from the Cosmic Ray Energetics And Mass for the International Space Station (ISS-CREAM) experiment
}

\author{
Eun-Suk Seo $a, b^{*}$ on behalf of the ISS-CREAM Collaboration \\ (a complete list of authors can be found at the end of the proceedings) \\ aInstitute for Physical Science and Technology, University of Maryland, \\ College Park, MD, USA \\ bDepartment of Physics, University of Maryland, \\ College Park, MD, USA \\ E-mail: seo@umd.edu
}

The Cosmic Ray Energetics And Mass for the International Space Station (ISS-CREAM) experiment took high-energy cosmic ray data for 539 days after its successful installation on the ISS in August 2017. The ISS-CREAM instrument is configured with complementary particle detectors capable of measuring elemental spectra for $Z=1-26$ nuclei in the energy range $10^{12}$ $10^{15} \mathrm{eV}$; as well as electrons at multi-TeV energies. The goal is to understand cosmic ray origin, acceleration, and propagation by extending direct measurements of cosmic rays to energies that overlap the energy region of air showers measurements. The four layers of finely segmented Silicon Charge Detectors provide precise charge measurements. They have been designed to minimize hits of accompanying backscattered particles in the same segment as the incident cosmic ray particle to avoid charge misidentification. The sampling tungsten/scintillating-fiber calorimeter, which is identical to the calorimeter for prior CREAM balloon flights, provides energy measurements. In addition, scintillator-based Top and Bottom Counting Detectors distinguish electrons from nuclei. Our analysis indicates that the data extend well above $100 \mathrm{TeV}$. Recent results from the ongoing analysis are presented.

37th International Cosmic Ray Conference (ICRC 2021)

July 12 th - 23rd, 2021

Online-Berlin, Germany 


\section{Introduction}

Indirect measurements from ground-based experiments have provided the all-particle spectrum from about $10^{14} \mathrm{eV}$ to $>10^{20} \mathrm{eV}$. These measurements have shown that the energy spectrum above $\sim 3 \times 10^{15} \mathrm{eV}$ is somewhat steeper than the spectrum below, forming the so-called spectral "knee". Whether and how the "knee" structure is related to the cosmic-ray acceleration and propagation are among the questions yet to be answered in particle astrophysics. The detailed energy dependence of elemental spectra at very high energies, where the rigidity-dependent supernova acceleration limit could be reflected in composition change, provides a key to understanding the origin, acceleration, and propagation of cosmic rays.

The Cosmic Ray Energetics And Mass (CREAM) experiment was initially developed to extend direct measurements of cosmic-ray elemental spectra to the highest energy practical in a series of balloon flights [1]. Following seven successful balloon flights in Antarctica with a cumulative exposure of 191 days, the CREAM payload was transformed for the International Space Station (ISS). This version of CREAM, aka ISS-CREAM, was launched aboard a SpaceX Falcon 9 rocket as part of the 12th Commercial Resupply Service (CRS-12) mission to the ISS on August 14, 2017. Two days after launch, the Dragon spacecraft with ISS-CREAM in the unpressurized trunk arrived at the ISS. The ISS-CREAM payload was extracted from the trunk and installed on the ISS JEM-EFU\#2. Following its successful activation on the ISS on August 22, 2017, high energy cosmic ray data were taken until February 12, 2019 for 539 days.

\section{ISS-CREAM Instrument}

To address whether there are any spectral differences among different elements and/or whether there are any spectral features that may be due to a supernova acceleration limit, simultaneous measurements of protons and heavier nuclei with a single instrument over a wide energy range are needed. The ISS-CREAM instrument is configured with complementary particle detectors to determine the charge and energy of high-energy cosmic rays from protons to iron nuclei over the energy range $\sim 10^{12}$ to $\sim 10^{15} \mathrm{eV}$ [2]. It is designed to meet the challenging requirement to have a large enough geometry factor to collect adequate statistics for the low flux of high-energy particles and yet stay within the JEM-EF weight and volume limit. The primary instrument flown on the balloon-borne CREAM was repackaged into the smaller volume available on the JEM-EF. The ISS-CREAM payload (Fig. 1 left) is about the size of a refrigerator, $1.85 \times 0.95 \times 1 \mathrm{~m}^{3}$, with a mass of $1,258 \mathrm{~kg}$.

As shown in Fig. 1 right, the ISS-CREAM instrument is configured with complementary particle detectors. Starting at the top, they include four layers of Silicon Charge Detector (SCD), carbon targets, the Top Counting Detector (TCD), the tungsten/scintillator sampling calorimeter (CAL), the Bottom Counting Detectors (BCD), and the Boronated Scintillator Detector (BSD). Both CAL, including carbon targets, and SCD were previously flown on balloons for energy and charge measurements, respectively. They have already demonstrated their capabilities of determining the energy and charge of high-energy cosmic rays from $10^{10}$ to $>10^{14} \mathrm{eV}$ for the proton to iron elemental range with excellent resolution [3].

The ISS-CREAM SCD is finely segmented to minimize the backscattered particles' contamination in the charge measurements. Each of the four SCD layers covers a $78 \times 74 \mathrm{~cm}^{2}$ 


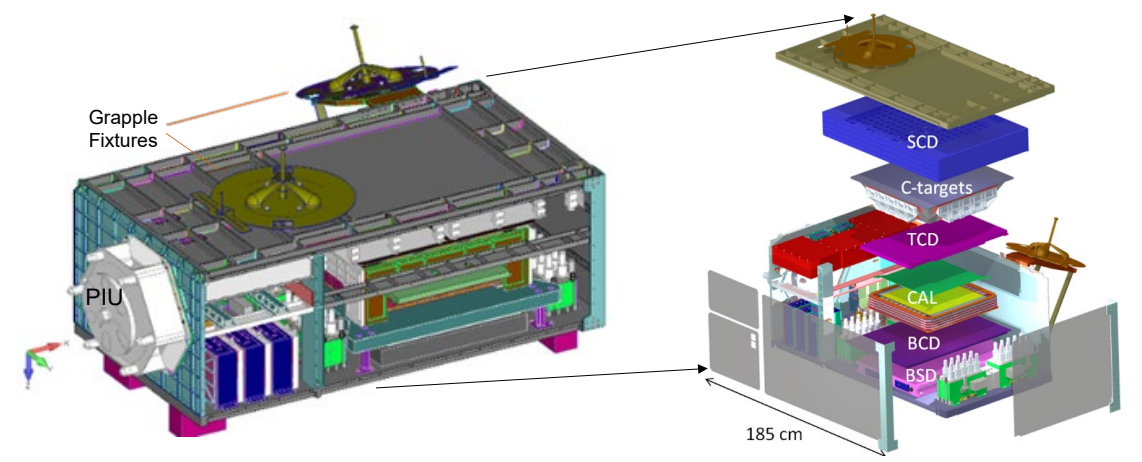

Figure 1. The ISS-CREAM payload configuration (left), and an exploded view of the ISSCREAM instrument (right). The white hexagon structure on the left is the Payload Interface Unit (PIU), which is the attachment point of the payload to the ISS JEM-EF. Two grapple fixtures are used by the ISS remote manipulator systems to transfer the payload from the launch vehicle to the ISS.

area. A cosmic ray passing through the sensor produces ionization that is proportional to the square of the particle charge. The $1.55 \times 1.38 \mathrm{~cm}^{2}$ active area of each pixel is optimized to reduce the effect of backscatter from showers in the calorimeter while keeping the channel count and power at manageable levels. Signals in sensors are readout with 16-channel CR-1.4A ASIC followed by 16-bit ADCs. This allows fine charge resolution over a wide dynamic range covering charges $Z=1$ to 26 [4].

The CAL combines $0.5 \lambda_{\text {int }}$ (interaction length) thick graphite targets and a stack of 20 tungsten plates with interspersed scintillating fiber layers. Each $50 \mathrm{~cm} \times 50 \mathrm{~cm} \times 3.5 \mathrm{~mm}(1$ radiation length thick) tungsten plate is followed by a layer of fifty scintillating fiber ribbons. Each $1 \mathrm{~cm}$-wide ribbon is made of nineteen $0.5 \mathrm{~mm}$ diameter scintillating fibers [10]. The carbon targets induce hadronic interactions so showers develop in the CAL rather uniformly. To cover a wide dynamic range, three (low, mid, and high) readout ranges are used via the optical division described in [5].

The TCD/BCD, based on plastic scintillators, are located above and below the CAL, respectively. Each of them is readout with $20 \times 20$ photodiode arrays. Each photodiode covers 2 $\mathrm{cm} \times 2 \mathrm{~cm}$ area. This segmentation helps electron measurements by distinguishing electrons from protons using different shower profiles between electrons and protons [6]. TCD/BCD also provide a low energy trigger while the CAL provides a high energy trigger. The Boronated Scintillator Detector (BSD) is located at the bottom of the instrument for additional e/p separation by measuring late thermal neutron shower activity [7].

\subsection{Calibrations}

As part of the extensive development program, the CREAM detectors were tested and calibrated at CERN's Super Proton Synchrotron (SPS), which provides the highest-energy testbeam particles available. These accelerator calibrations were essential for both functional verification of the detectors and for verification of the simulation model, which must be relied on extrapolating the accelerator response to the response for much-higher-energy cosmic rays observed during the flight exposures.

As discussed in Zhang et al.[8], and references therein, the particle beam data are in good agreement with detailed Monte Carlo simulations. The instrument was also exposed to $\mathrm{A} / \mathrm{Z}=2$ 
nuclear fragments of the $158 \mathrm{GeV} /$ nucleon indium beam at CERN. The energy deposit as a function of mass number shows good linearity for $158 \mathrm{GeV}$ to $\sim 9 \mathrm{TeV}$ incident energy $[9,10]$. The simulations show that the calorimeter response is quite linear and that its resolution is nearly energy independent up to $10^{15} \mathrm{eV}$, where the experiment is limited by low particle fluxes.

The in-flight calibration included periodic charge injection events, LED flashes, and pedestal runs to provide the information needed to correct for electronics including gain changes as a function of time, e.g., due to changes in temperature, etc. It is important to take into account any instrument setting differences between the beam-test calibration on the ground and the flight operations.

\subsection{Flight Operations}

The command uplink and data downlink for the ISS data are through the Tracking and Data Relay Satellite System (TDRSS), exactly analogous to balloon-borne CREAM operations. The Science Operations Center (SOC) established at the University of Maryland for the past seven balloon flights was utilized for the ISS-CREAM operation. The SOC's operation was coordinated with the Huntsville Operations Support Center (HOSC)/Payload Operations Integration Center (POIC) at NASA's Marshall Space Flight Center.

The CREAM Data Acquisition system (CDAQ) utilizes the Software Toolkit for Ethernet Lab-Like Architecture (STELLA) for the communications between the SOC and the ISS-CREAM payload. The Telescience Resources Kit (TRek) was used for data/command path between HOSC and SOC.

The Science Flight Computer (SFC)'s functional status was monitored using two watchdog timers, and the instrument-interface unit took necessary actions autonomously when it was needed, e.g., rebooting, selecting boot-source, and power switching between two SFC's. CDAQ commands could also be sent to ISS-CREAM through a timed sequence called a time-liner, which made the SOC operation rather autonomous [11].

\section{Data Analysis}

Over 60 million cosmic-ray events were collected with the ISS-CREAM instrument. At least one hit in each of TCD and BCD provides a coincidence trigger. The calorimeter trigger is designed for high energy shower events by requiring significant signals in a number of consecutive layers (changeable by commands but typically six was used in flight). For an event selected with a science trigger condition, its trajectory was reconstructed from a linear fit to the core of the shower axis through the multiple layers of scintillating fiber ribbons in $\mathrm{CAL}$ as illustrated in Fig. $2[12,13]$. An extrapolation of this reconstructed trajectory was required to traverse the active areas of both the SCD and the bottom of the CAL. The silicon pixel at the

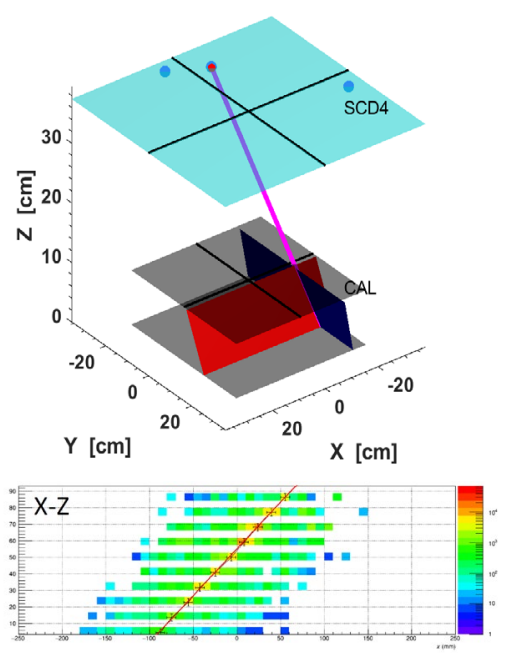

Figure 2. An illustration of a CAL track extrapolated to the SCD using Monte Carlo simulation (top) and a flight data example showing a shower developed in $C A L$. The red line is a linear fit to the shower axis (bottom). 
extrapolated position was selected for the charge measurement. The signal in that pixel was corrected for the incident angle before making the charge determination. As shown in Fig. 3, individual elements from $Z=1$ to 26 were identified with $\sigma \sim 0.2 \mathrm{e}$ charge resolution and good linearity. The relative abundance in this plot has no physical significance because needed corrections for interactions and propagation have not been applied to these data $[14,15]$.

The incident cosmic-ray particle energy was determined from ionization energy deposits of cascades developed in the CAL. The calorimeter energy deconvolution included corrections for both the small energy dependence of the shower leakage and the energy resolution, as described in [16].

The measured spectra were corrected for background from misidentified charges due to interactions above the charge detector and the effect of particles backscattered from the calorimeter. The absolute flux was obtained by correcting the measured spectra for the trigger, reconstruction and event selection efficiencies, the geometry factor, and live time. Statistical uncertainties were estimated with $84 \%$ Poisson confidence limit for the highest energy bins, where the number of particles is less than 10 . The spectral analysis procedures with ISS-CREAM data generally follow the same procedures used in analyzing the balloon-borne CREAM data. Event selection, charge determination, energy measurements, spectral deconvolution, background corrections, absolute fluxes, and uncertainties were handled in a manner comparable to previous work [16, and references therein]. However, the correction for the atmospheric overburden was not needed for the ISS-CREAM data. More details of the current analysis on the ISS-CREAM spectral measurements can be found in $[14,15]$.

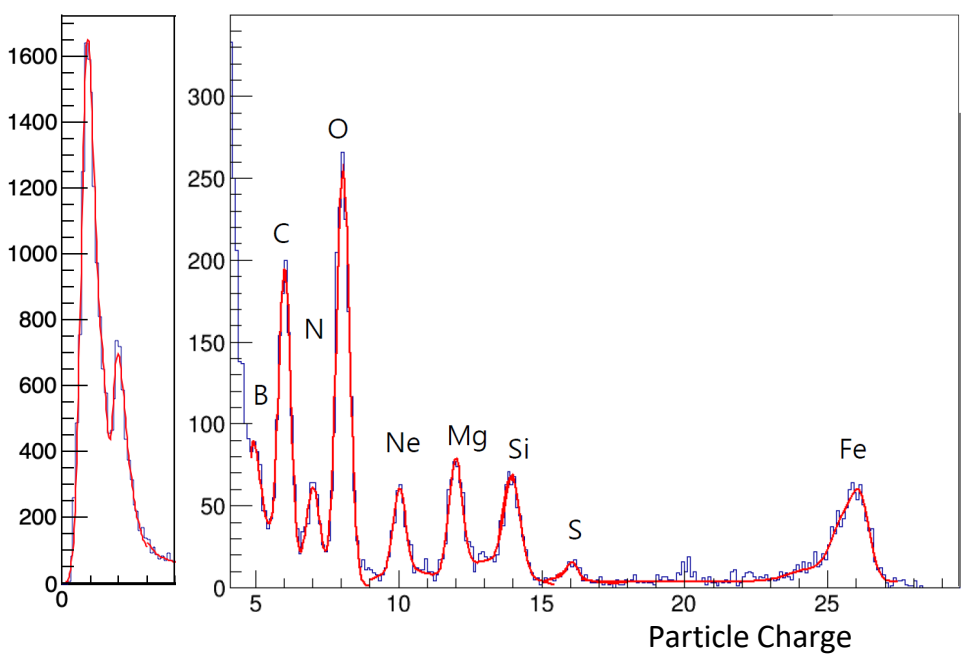

Figure 3. Charge distribution measured with the ISS-CREAM instrument during the flight.

\section{Results \& Discussions}

The ISS-CREAM proton spectrum in the energy range $2.5 \times 10^{12}-6.5 \times 10^{14} \mathrm{eV}$ is shown in Fig. 4. The flux is multiplied by $E^{2.7}$ for spectral features to be easily seen. Only the statistical uncertainties are shown as error bars. See [14] for detailed discussion on uncertainties including systematic uncertainties. The ISS-CREAM data are compared with selected previous measurements: Alpha Magnetic Spectrometer (AMS-02), Advanced Thin Ionization Calorimeter 
(ATIC), Calorimetric Electron Telescope (CALET), the balloon-borne CREAM, DArk Matter Particle Explorer (DAMPE), Payload for Antimatter-Matter Exploration and Light-nuclei Astrophysics (PAMELA), and NUCLEON [17, and references therein]. The ISS-CREAM proton spectrum is in agreement with previous measurements where they overlap.

Our data deviate from a single power law and confirm the spectral softening near $10 \mathrm{TeV}$ reported in the prior balloon-borne CREAM, DAMPE, and NUCLEON. A broken power law fit to $2.5-100 \mathrm{TeV}$ data gives an index of $\gamma=2.65 \pm 0.06$ for a power law $\mathrm{E}^{-\gamma}$ with a break at $\sim 9.94$ $\pm 4.6 \mathrm{TeV}$ and $\Delta \gamma=0.26 \pm 0.1$. At higher energies, the softening does not continue but the spectrum becomes harder again.

The compiled proton data are also compared with calculated curves in Figure 4. Curves illustrate the cosmic-ray spectrum from four different classes of sources [18], each indicating a power law in rigidity with its specific spectral index and a simple rigidity (momentum per charge) dependent acceleration limit: $E_{\max _{-} z}=Z \times E_{\max _{\_} \text {, where }} Z$ is charge, $E_{\max _{-} z}$ is the acceleration limit for charge $Z$ particles, and $E_{\max \_p}$ is the acceleration limit for protons. This calculation is similar to the work of [19], but a low energy class is added to reflect the spectral hardening at $200 \mathrm{GV}$. Each dashed curve presents a power law spectrum with a rigidity cutoff: (1) the orange dashed curve for $\sim \mathrm{R}^{-2.9}$ with a cutoff at $400 \mathrm{GV}$, (2) the red dashed curve for $\sim \mathrm{R}^{-2.5}$ with a cutoff at $50 \mathrm{TV}$, (3) the blue dashed curve $\sim \mathrm{R}^{-2.5}$ with a cutoff at $4 \mathrm{PV}$, and (4) the cyan dashed curve for $\sim \mathrm{R}^{-2.2} \mathrm{a}$ cutoff at $500 \mathrm{PV}$. The green solid curve is the sum of all four. It illustrates both the spectral hardening at $\sim 200 \mathrm{GV}$ and the spectral softening at $\sim 10 \mathrm{TV}$ could be due to a transition from one source class to another. Although not shown in this paper, the all-particle spectrum calculated by summing up major elements with the same method is consistent with the measured "knee" at $\sim 3$ $\mathrm{x} 10^{15} \mathrm{eV}$ as demonstrated in [18].

It should be mentioned that Zatsepin and Sokolskaya [20] proposed three classes: one from the explosion of isolated stars into the interstellar medium, another one from supernovae within the local superbubble, and the other one from nova stars with consideration of solar modulation

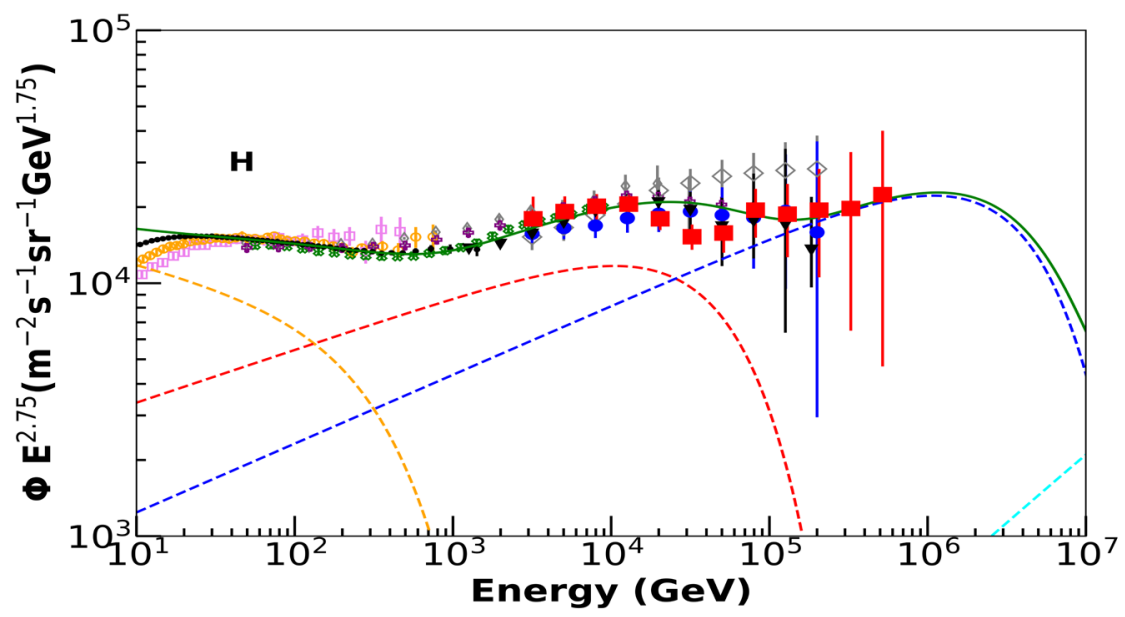

Figure 4. The ISS-CREAM proton spectrum (red filled squares) is compared with previous experiments: small black circles, AMS-02; pink open squares, BESS; orange open circles, PAMELA; grey narrow diamonds, ATIC; blue filled circles, CREAM-I; black downward triangles, CREAM-III; purple crosses, DAMPE; green X's, CALET; and grey diamonds, NUCLEON. Curves are described in the main text. 
effects characterized by the modulation parameter. Ptuskin et al. [21] considered different types of supernova remnants (SNRs) and their evolution, including Type Ia, Type IIP, Type Ib/c, and Type IIb SNRs. Effects of reverse shocks and modified shocks from interactions with cosmic ray particles could generate a concave source spectrum. See [22] for an example of other attempts to explain the observed spectra with breaks in the source spectra or/and diffusion coefficient.

\section{Conclusions}

The ISS-CREAM instrument successfully collected high energy cosmic ray data between August 14, 2017 and February 12, 2019. The newly developed TCD/BCD provided low energy trigger and finely segmented SCD distinguished individual elements with excellent charge resolution. CAL provided energy measurements, tracking, and a high energy trigger. The resulting proton spectrum confirms the spectral softening at $\sim 10 \mathrm{TeV}$, which may indicate a transition from one source class to another. This bump-like structure is consistent with the deviation from a single power law reported by CREAM-I \&III, DAMPE, and NUCLEON, but ISS-CREAM extends measurements to higher energies than those prior measurements.

The observed deviation from a single power law challenges the traditional propagation models. In principle, proposed explanations for spectral hardening at $\sim 200 \mathrm{GV}$ could be applied to the softening at $\sim 10 \mathrm{TeV}$ with breaks in the source spectra and/or diffusion coefficient reflecting nonlinear effects in diffusive shock acceleration and/or spatial variations in diffusion properties. However, it might be more natural to assume several source populations dominant at different energies.

In addition to the most abundant protons, other heavier $Z \geq 2$ elements are being analyzed. Simultaneous measurements of elemental spectra of $Z=1-26$ nuclei will be important for a coherent model development to understand cosmic ray origin, acceleration, and propagation.

\section{Acknowledgements}

This work was supported in the U.S. by NASA grant NNX17AB41G, in Korea by MSIT under the High-Potential Individuals Global Training Program (2021-0-01544) supervised by the IITP and National Research Foundation grants 2018R1A6A1A06024970, 2017K1A4A3015188 and 2021R1A2B5B03002645, and their predecessor grants. It was also supported in France by IN2P3/CNRS and CNES and in Mexico by DGAPA-UNAM project IN109617. The authors thank NASA GSFC WFF and its contractors for engineering support and project management, JSC ISS Program Office for the launch support and ISS accommodation, MSFC for the operational support, and KSC and SpaceX for the launch support.

\section{References}

[1] E. S. Seo et al., Cosmic-ray energetics and mass (CREAM) balloon project, Adv. in Space Res. 33/10 (2004) 1777.

[2] E. S. Seo et al. Cosmic Ray Energetics And Mass for the International Space Station (ISS-CREAM), Adv. in Space Res. 53/10 (2014) 1451.

[3] H. S. Ahn et al. Discrepant Hardening Observed in Cosmic-ray Elemental Spectra, Astrophys. J. Lett. 714 (2010) L89. 
[4] J. Lee et al. The ISS-CREAM Silicon Charge Detector for identification of the charge of cosmic rays up to $Z=26$ : design, fabrication and ground-test performance, Astropart. Phys. 112 (2019) 8.

[5] H. S. Ahn et al. The Cosmic Ray Energetics And Mass (CREAM) instrument, Nucl. Inst. Meth. A 579 (2007) 1034.

[6] S. C. Kang et al. On-orbit performance of the Top and Bottom Counting Detectors for the ISSCREAM Experiment on the International Space Station, Adv. in Space Res. 64/12 (2019) 2564.

[7] Y. Amare et al. The Boronated Scintillator Detector of the ISS-CREAM Experiment, Nucl. Instrum. Methods A 943 (2019) 162413.

[8] H. G. Zhang et al. Performance of the ISS-CREAM calorimeter in a calibration beam test, Astropart. Phys. 130 (2021) 102583.

[9] H. S. Ahn et al. Performance of CREAM Calorimeter: Results of Beam Tests, Nuclear Physics B(Poc. Suppl.) 150 (2006) 272.

[10] I. H. Park et al. Heavy ion beam test results of the silicon charge detector for the CREAM cosmic ray balloon mission. Nucl. Instrum. Meth. A 535 (2004) 158.

[11] K. C. Kim et al. ISS-CREAM Flight Operations, PoS(ICRC2019)087.

[12] P. Lundquist et al. Track Reconstruction for ISS-CREAM Resulting in Improved Energy and Charge Resolution, PoS(ICRC2019)099.

[13] R. Takeishi et al. Cosmic-Ray Elemental Spectra Measured With ISS-CREAM, PoS(ICRC2019)140.

[14] G. H. Choi et al. ISS-CREAM Collaboration, Analysis Result of the High-Energy Cosmic-Ray Proton Spectrum from the ISS-CREAM Experiment, POS(ICRC2021)094.

[15] S.C. Kang et al. ISS-CREAM Collaboration, Cosmic-ray Heavy Nuclei Spectra Using the ISSCREAM Instrument, POS(ICRC2021)097.

[16] Y. S. Yoon et al. Proton and Helium Spectra from the CREAM-III Fight, Astrophys. J. 839 (2017) 5.

[17] E. S. Seo, Advances in Direct Measurements of Cosmic Rays, J. Korean Phys. Soc., 78/10 (2021) 923.

[18] R. Scrandis, D. P. Bowman and E. S. Seo, Cosmic Ray Elemental Spectra and Atmospheric Neutrino fluxes, PoS(ICRC2021)1220.

[19] T. K. Gaisser, T. Stanev and S. Tilav, Cosmic Ray Energy Spectrum from Measurements of Air Showers, Astropart. Phys. 35 (2012) 801.

[20] V. I., Zatsepin and N. V. Sokolskaya, Three component model of cosmic ray spectra from $10 \mathrm{GeV}$ to $100 \mathrm{PeV}, \mathrm{A} \& \mathrm{~A}, 458$ (2006) 1.

[21] V. S. Ptuskin, V.N., Zirakashvili, and E.S., Seo, Spectra of Cosmic-Ray Protons and Helium Produced in Supernova Remnants, Astrophys. J. 763 (2013) 47.

[22] H. Wu, E.S. Seo and V. Ptuskin, Study Of Cosmic Ray Spectral Hardening Using GALPROP, POS(ICRC2021)155. 


\section{Full Authors List: ISS-CREAM Collaboration}

S. Aggarwal ${ }^{\mathrm{a}, \mathrm{b}}$, Y. Amare ${ }^{\mathrm{a}}$, D. Angelaszek ${ }^{\mathrm{a}, \mathrm{b}}$, D. P. Bowman, ${ }^{\mathrm{b}}$ Y. C. Chen ${ }^{\mathrm{a}, \mathrm{b}}$, G. H. Choi ${ }^{\mathrm{c}}$, M. Copley ${ }^{\mathrm{a}}$, L. Derome ${ }^{\mathrm{d}}$, L. Eraud ${ }^{\mathrm{d}}$, C. Falana $^{\mathrm{a}}$, A. Gerrety ${ }^{\mathrm{a}}$, J. H. Han ${ }^{\mathrm{a}}$, H. G. Huh ${ }^{\mathrm{a}}$, A. Haque ${ }^{\mathrm{a}, \mathrm{b}}$, Y. S. Hwang ${ }^{\mathrm{e}}$, H. J. Hyun ${ }^{\mathrm{e}}$, H.B. Jeon ${ }^{\mathrm{e}}$, J. A. Jeon ${ }^{\mathrm{c}}$, S. Jeong ${ }^{\mathrm{c}}$, S. C. Kang, ${ }^{\mathrm{e}}$ H.

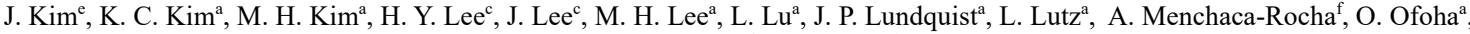
H. Park ${ }^{\mathrm{e}}$, I. H. Park ${ }^{\mathrm{c}}$, J. M. Park ${ }^{\mathrm{e}}$, N. Picot-Clemente ${ }^{\mathrm{a}}$, R. Scrandis ${ }^{\mathrm{a} . b}$, E. S. Seo ${ }^{\mathrm{a}, \mathrm{b}}$, J. R. Smith ${ }^{\mathrm{a}}$, R. Takeishic ${ }^{\mathrm{c}}$ P. Walpole ${ }^{\mathrm{a}}$, R. P. Weinmann ${ }^{\mathrm{a}}$, H. Wu ${ }^{\mathrm{a}, \mathrm{b}}$, J. Wu $\mathrm{W}^{\mathrm{a}}$ Z. Yin ${ }^{\mathrm{a}, \mathrm{b}}$, Y. S. Yoon ${ }^{\mathrm{a}, \mathrm{b}}$ and H. G. Zhang

${ }^{a}$ Inst. for Phys. Sci. and Tech., University of Maryland, College Park, MD, USA

${ }^{b}$ Dept. of Physics, University of Maryland, College Park, MD, USA

${ }^{c}$ Dept. of Physics, Sungkyunkwan University, Republic of Korea

${ }^{d}$ Laboratoire de Physique Subatomique et de Cosmologie, Grenoble, France

${ }^{e}$ Dept. of Physics, Kyungpook National University, Republic of Korea

${ }^{f}$ Instituto de Fisica, Universidad Nacional Autonoma de Mexico, Mexico 